phyrin cores, with pyrrole rings alternately located above and below the porphyrin planes. These rings were linked by the trinuclear bridging of $\mathrm{Co}(\mathrm{II})$ carboxylate clusters. PIZA-1 has oval-shaped channels of $9 \AA \times 7 \AA$ along the $b$ and $c$ axes, with a $14 \AA \times 7 \AA$ channel along the $a$ axis. Thermal analysis demonstrated a $60 \%$ mass loss associated with solvent molecules and coordinated pyridine and water molecules.

PIZA-1 demonstrated unique properties attributed to its hydrophilic cavity sites, $74 \%$ void space, and overall neutrality and robustness. In particular, PIZA-1 showed the ability to absorb high amounts of polar solvents. The researchers reported that 162 water molecules per unit cell (which has four metalloporphyrins) could be absorbed, and in a 24-h time frame, $105 \mathrm{ml} / 100 \mathrm{~g}$ was absorbed compared with $22 \mathrm{ml} / 100 \mathrm{~g}$ for traditional desiccant zeolites (e.g., Linde 4A). The researchers found similar results for amines and alcohols, which showed steric and chain-length dependence.

PIZA-1 demonstrated the ability to dry organic solvents. In this case, thiol, nitrile, aldehyde, bromide, toluene, benzene, tetrahydrofuran, and hexane may be used, as they showed little absorption. With their ability to change the metal centers and porphyrin functionalities, such structures may open new avenues in catalysis and further selective separations.

MATHEW M. MAYE

\section{Self-Assembled, Surface-Induced Nanoscale Patterns Observed in Langmuir-Blodgett Films}

Formation of surface-induced nanoscale patterns in polymer layers is the subject of recent intense experimental and theoretical studies. In the October issue of Nano Letters, A.L. Simões Gamboa, E.J.M. Filipe, and P. Brogueira from Instituto Superior Técnico in Portugal have reported their approach to prepare nanoscale periodic structures on the surface with potentially low surface energy, playing with the self-assembling capabilities of a diblock copolymer and a short diblock molecule, a semifluorinated $n$-alkane oligomer, $\mathrm{F}\left(\mathrm{CF}_{2}\right)_{n}\left(\mathrm{CH}_{2}\right)_{m} \mathrm{H}$.

The use of semifluorinated $n$-alkanes (SFAs) as side groups in homopolymers or block copolymers has been previously shown to generate polymers with low-surface-energy properties. On the other hand, semifluorinated $n$-alkanes have been reported to spread at the air-water interface and form Langmuir films. In the present approach, the semifluorinated $n$-alkane $\mathrm{F}\left(\mathrm{CF}_{2}\right)_{8}\left(\mathrm{CH}_{2}\right)_{18} \mathrm{H}$ was "blended" with an amphiphilic block copolymer, and the blend was confined at the air-water interface by the Langmuir technique. To do so, mixed solutions, corresponding to various proportions of SFAs and a polystyrene-poly(ethylene oxide) (PSPEO) diblock copolymer in chloroform, were spread at the surface of deionized water in a Langmuir trough; the spread films were then compressed. The PS-PEO copolymer used had a PS block of 40 monomers and a PEO block of 123 monomers. The hydrophobic PS blocks graft the copolymer to the air-water interface; the PEO blocks are adsorbed at the interface at a low number of grafts per unit area and stretched into the water subphase as the film is compressed, eventually resulting in a "brush" configuration. The films were transferred onto hydrophilic glass substrates by the Langmuir-Blodgett technique at desired polymer grafting densities.

From the surface pressure versus area curves for the prepared Langmuir films, the researchers observed that the presence of SFAs has a significant effect on the films: The maximum surface pressure obtained by compressing the film increases with increasing PS-PEO grafting density. But all of the films' surface pressure versus area curves have a shape that resembles the curve of pure SFAs and will reach about the same limiting area value as the pure SFA film. Consequently, the surface will be

\section{Fast And Contact-Free}

The LSM 5 Pascal Laser Scanning Microscope efficiently analyzes microstructures. Precision optics, innovative scanning strategies \& powerful, modular software packages guarantee maximum operating convenience and flexibility. Scanning field can be expanded by 3D stitching.

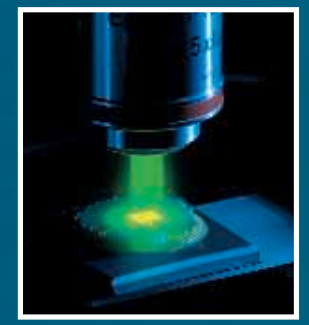

For more information, call 800-233-2343.

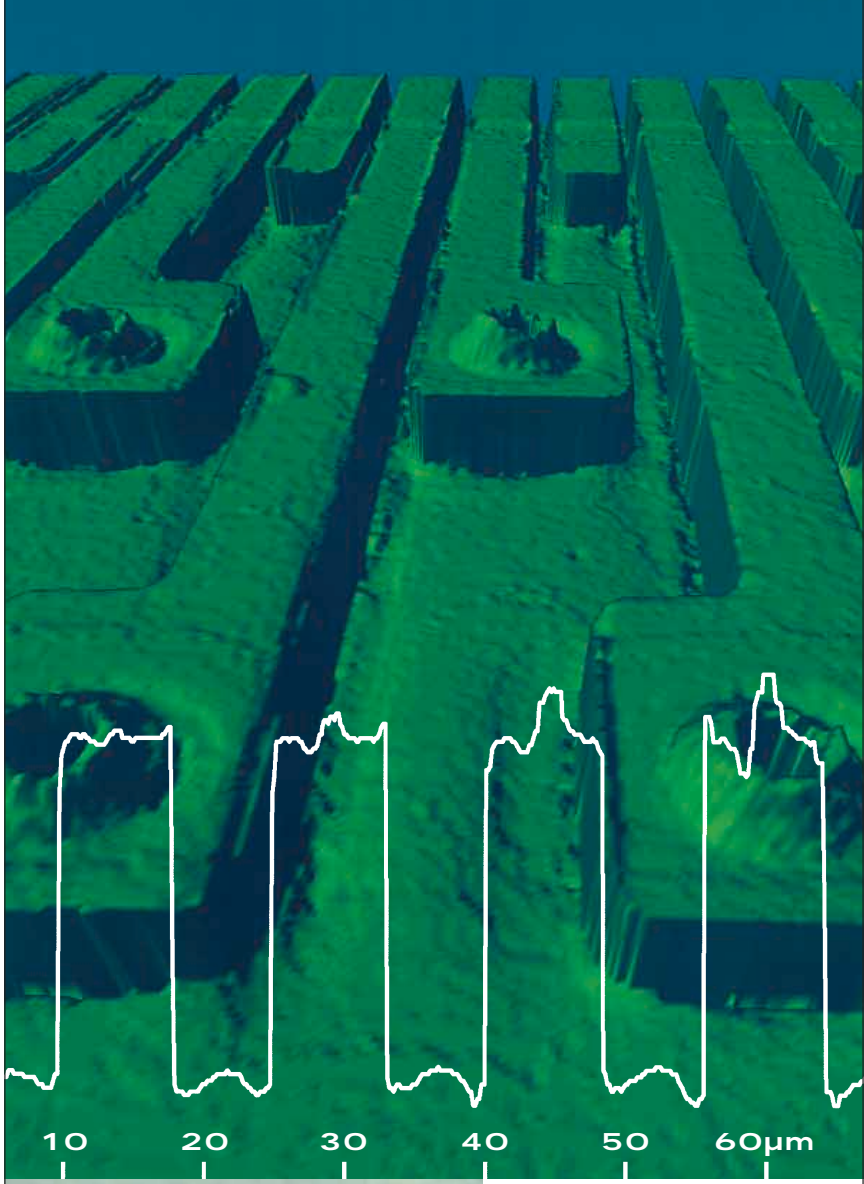

Carl Zeiss Microlmaging, Inc Thornwood, NY 10594 micro@zeiss.com zeiss.com/lsm-mat

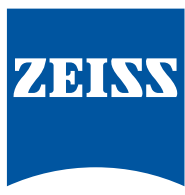

We make it visible.

Circle No. 37 on Inside Back Cover 


\section{The $F$ ischerscope ${ }^{\circledR} \mathrm{H} 100$ \\ A II the Right Properties...}

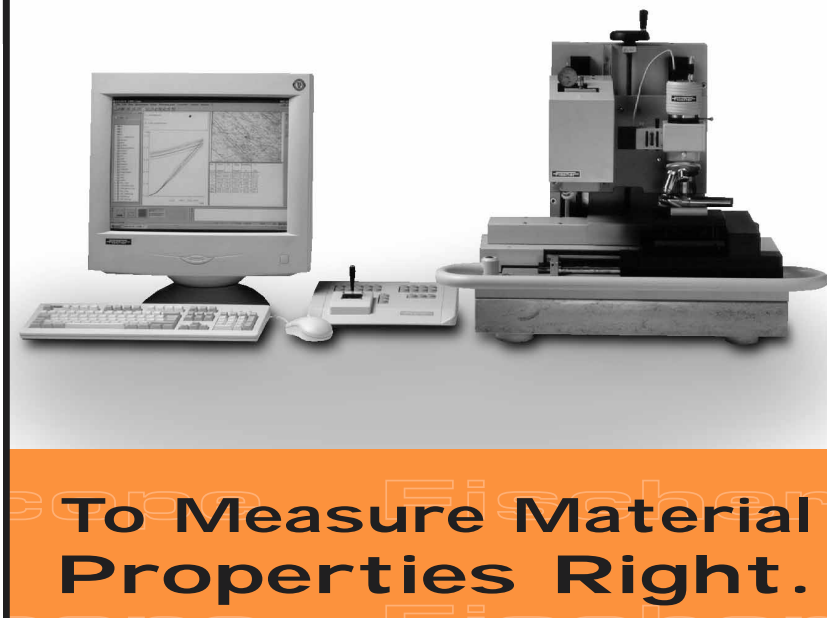

Measuring microhardness has never been easier with the innovative Fischerscope ${ }^{\circledR} \mathrm{H100}$ ! Testing for microhardness and material properties under ultra-low load, the H100 leaves virtually no visible indentation traces on the testing surface. $\square$ Extremely smart and sensitive, the $\mathrm{H} 100$ is driven by specially developed Windows ${ }^{\circledR}$ based software. It offers a full spectrum of material property values, including surface and hardness profiles, creep behavior data, dynamic indentation measurements, and viscoelastic properties, including Young's modulus. $\square$ The H100 automatically calculates results, eliminating the need for subjective measurements that could compromise results. The Fischerscope $\mathrm{H} 100$ is ideal for evaluating virtually any material from soft elastomers to ultra-hard tool coatings. For material research and a host of other applications the H100 has All the right properties to measure material properties right.

\section{1 -8 00 -2 43 -8 417}

w w w. fis c her-tec hnology. com

Fischer Technology, Inc.

750 Marshall Phelps Road - Windsor, CT 06095 Phone: 860-683-0781 - Fax: 860-688-8496 enriched SFA. The SFA vertically segregates to the top of the PSPEO layer at the air-water interface.

The mixed films were characterized by atomic force microscopy (AFM), revealing nanoscale periodic structures. The AFM images showed that their surface structures are honeycomb-like, having a hump at the center with a periodicity of about $40 \mathrm{~nm}$. The same pattern was shown in all films regardless of the polymer grafting density, but the ordering increased with increasing polymer grafting density.

Although the researchers prepared Langmuir-Blodgett films of a SFA and a PS-PEO diblock copolymer, more detailed structural analysis, such as the composition of the humps and honeycomb walls, and a systematic route to prepare this structured material need to be further investigated.

Yue Hu

\section{Ancient Rust-Proof Iron Pillar Possibly Protected by Layer of "Misawite" ( $\delta$-FeOOH)}

A 1600-year-old wrought-iron pillar that stands near New Delhi, India, as a well-known landmark and tourist site has intrigued metallurgists and historians for centuries. Subjected to heat, humidity, and even a direct cannonball hit, the pillar has remained essentially rust-proof. Several theories have been suggested for its virtually rust-free condition. The mystery now appears to have been solved due to the work of metallurgist R. Balasubramaniam from the Indian Institute of Technology, Kanpur.

The Delhi iron pillar was built in the 4th century AD, and stands $23 \mathrm{ft}$ tall with a diameter of $16.5 \mathrm{in}$. at the bottom and $12.5 \mathrm{in}$. at the top. It is estimated to weigh $\sim 6.5$ tons. It is considered to be the biggest hand-forged block of iron from antiquity. In an article published in the November issue of Current Science, Balasubramaniam demonstrates that the lack of rust is due to the formation of a protective layer of misawite $(\delta-\mathrm{FeOOH})$, as well as a layer of crystalline phosphates, catalyzed by the presence of comparatively high levels of phosphorus in the iron.

Rust samples from the pillar have been analyzed using x-ray diffraction, Fourier transform infrared spectroscopy, and Mossbauer spectroscopy. The samples were found to contain amorphous oxyhydroxides, including lepidocrocite $(\gamma-\mathrm{FeOOH})$, goethite $(\alpha-\mathrm{FeOOH})$ and misawite $(\delta$-FeOOH$)$, magnetite $\left(\mathrm{Fe}_{3-x} \mathrm{O}_{4}\right)$, and crystalline phosphates, mainly iron hydrogen phosphate hydrate $\left(\mathrm{FePO}_{4} \bullet \mathrm{H}_{3} \mathrm{PO}_{4}\left(4 \mathrm{H}_{2} \mathrm{O}\right)\right)$.

The initial corrosion of the iron, aided by slag inclusions, forms lepidocrocite and goethite, which enhances the concentration of $\mathrm{P}$ $(>0.1 \%$ in the base metal) at the metal-scale interface. This catalyzes the formation of amorphous misawite $(\delta-\mathrm{FeOOH})$, which offers significant initial corrosion resistance. In addition, the $\mathrm{P}$ reacts with moisture and forms phosphoric acid, which leads to the precipitation of amorphous phosphates at the metal-oxide interface. The phosphates, which form a thin continuous layer next to the metal, minimize corrosion because of their inhibitive nature. Over time, the amorphous phosphates transform to a crystalline phase due to alternate wetting and drying cycles. This further enhances the corrosion resistance due to the low porosity and compactness of the crystalline phase.

Kinetics studies show that an initial fast corrosion rate occurs for about three years until the misawite layer is formed. Subsequently, the corrosion rate is negligible, with corrosion resistance lasting for centuries.

Gopal RAO

\section{Decrystallization, Magnetic-Property Changes Caused at Sub-Melting-Point Temperatures Using Microwave Processing}

Crystalline to noncrystalline transformations in a bulk material typically require an intermediate liquid or gaseous state. Now, 